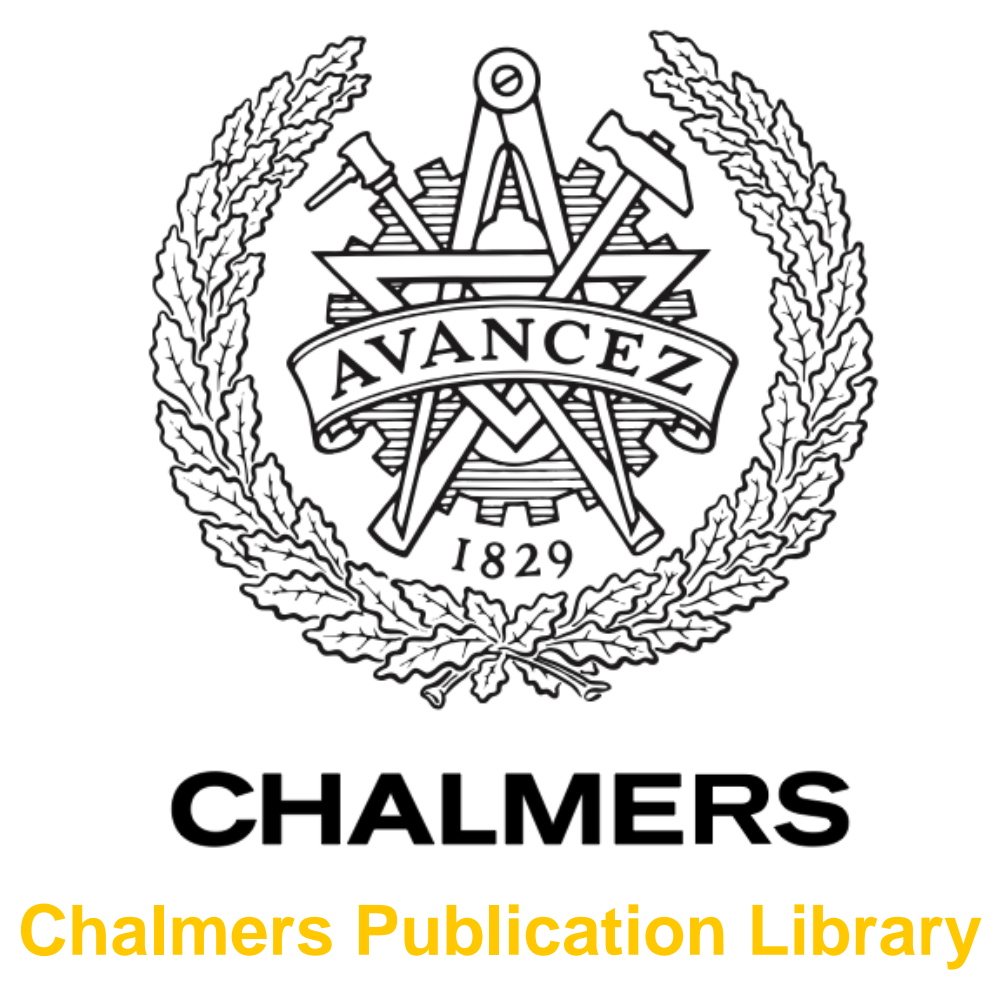

\title{
Investigation of penetration ability of UWB antennas in near-field sensing applications
}

This document has been downloaded from Chalmers Publication Library (CPL). It is the author's version of a work that was accepted for publication in:

Proceedings of 6th European Conference on Antennas and Propagation, EuCAP 2012. Prague, 26-30 March 2012

Citation for the published paper:

Razavi, A. ; Yang, J. (2012) "Investigation of penetration ability of UWB antennas in nearfield sensing applications". Proceedings of 6th European Conference on Antennas and Propagation, EuCAP 2012. Prague, 26-30 March 2012 pp. 791-795.

http://dx.doi.org/10.1109/EuCAP.2012.6205888

Downloaded from: http://publications.lib.chalmers.se/publication/156674

Notice: Changes introduced as a result of publishing processes such as copy-editing and formatting may not be reflected in this document. For a definitive version of this work, please refer to the published source. Please note that access to the published version might require a subscription.

Chalmers Publication Library (CPL) offers the possibility of retrieving research publications produced at Chalmers University of Technology. It covers all types of publications: articles, dissertations, licentiate theses, masters theses, conference papers, reports etc. Since 2006 it is the official tool for Chalmers official publication statistics. To ensure that Chalmers research results are disseminated as widely as possible, an Open Access Policy has been adopted.

The CPL service is administrated and maintained by Chalmers Library. 


\title{
Investigation of Penetration Ability of UWB Antennas in Near-field Sensing Applications
}

\author{
Aidin Razavi and Jian Yang \\ Dept. of Signals and Systems \\ Chalmers University of Technology \\ Gothenburg, Sweden \\ aidin.razavi@chalmers.se; jian.yang@chalmers.se
}

\begin{abstract}
Characterization of antennas in far-field applications, both in Line-Of-Sight (LOS) applications (such as in radar systems, point-to-point communications, satellite communications, radio telescopes, etc.) and multipath environment applications (such as mobile communications, indoor communications and indoor geolocations, etc.), is well defined and can be evaluated by well-developed measurement techniques. However, it is still not established how to characterize antennas in nearfield sensing applications, where most of the characteristics of antenna in far-field applications are not valid anymore. In this paper, we investigate the characteristics of antennas in nearfield sensing applications. The penetration ability, both in timedomain and in frequency-domain is introduced and evaluated. Three UWB antennas, with both simulated and measured results are presented here to demonstrate and evaluate the penetration ability, and the difference from the far-field characteristics, such as the directivity. It is concluded that the higher directivity in far-field does not guarantee a deeper penetration in near-field.
\end{abstract}

Index Terms-ultra-wideband antenna system, shape reconstruction.

\section{INTRODUCTION}

In recent years the interest in ultra-wideband (UWB) technology and therefore UWB antennas has increased. Large number of new UWB antenna designs have been presented in literature. Many of these antennas are supposed to be used in near-field applications (such as biomedical imaging, sensing, product quality assessment, etc.). Characterization of antennas in far-field applications, both in Line-Of-Sight (LOS) applications (such as in radar systems, point-to-point communications, satellite communications, radio telescopes, etc.) and multipath environment applications (such as mobile communications, indoor communications and indoor geolocations, etc.), is well defined and can be evaluated by well-developed measurement techniques [1]-[3]. However, characterization of antennas in near-field applications is not as well-defined and established as in far-field applications. Characteristics, such as radiation pattern, directivity, gain and radiation efficiency, are defined for far-field applications and not very proper anymore in near-field applications.

The purpose of this work is to investigate the penetration ability of UWB antennas through lossy materials, as one characteristic of UWB antennas in near-field applications.

Three UWB antennas, self-grounded Bow-Tie antenna [4], [5], Vivaldi antenna [6] and Antipodal Vivaldi antenna [7],

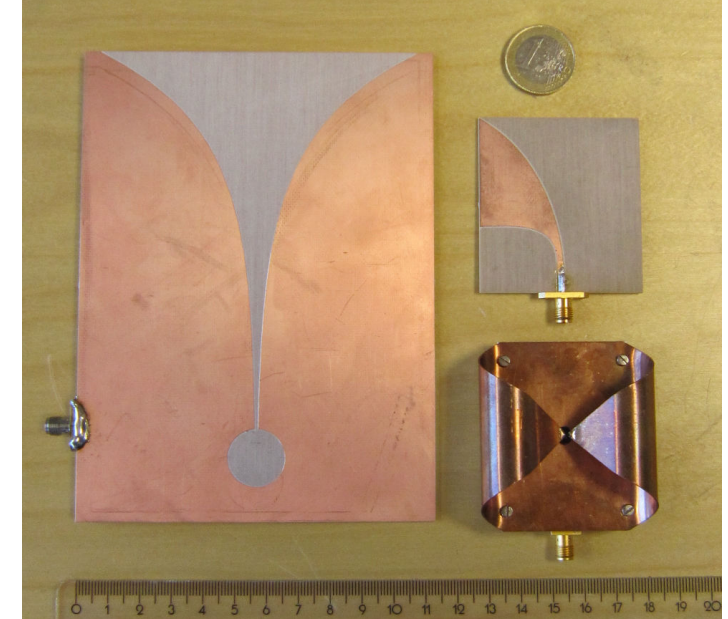

Fig. 1: Three UWB antennas investigated in this work: Vivaldi (left), Antipodal Vivaldi (top right) and Self-grounded BowTie (bottom right)

which are quite representative of UWB antennas, have been investigated to demonstrate and evaluate their penetration ability, based on both the simulated and measured results. Photos of these antennas are shown in Fig. 1. Notice the size difference of antennas.

It is observed that the higher directivity in far-field does not guarantee a deeper penetration in near-field.

\section{Definitions}

A general geometry of a through-sensing system can be presented by Fig. 2. Two antennas (or sensors) are positioned on the opposite sides of an Area Under Detection (AUD). One antenna transmits pulses while the one on the other side acts as a receiver for pulses propagated through the AUD. In this paper, we consider only the two antennas being in-line with each other and also touching the surface of AUD. (i.e. $D_{\text {air }}=0$ and $S=0$ ).

The total penetration coefficient $p_{t o t T S}$ for throughsensing (TS) system is defined as

$$
p_{t o t T S}=\frac{P_{r e c}}{P_{\text {in }}},
$$




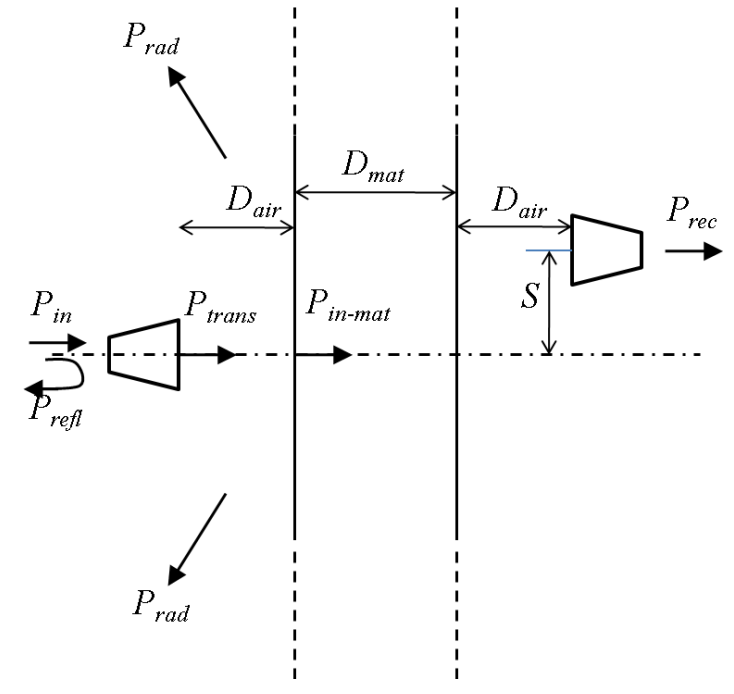

Fig. 2: Through sensing system.

where $P_{\text {rec }}$ and $P_{\text {in }}$ are the received power by receiving antenna and the input power at the transmitting antenna, respectively.

The impedance-match efficiency $e_{i m p}$ at the input port of the transmitting sensor is defined as

$$
e_{i m p}=\frac{P_{\text {trans }}}{P_{\text {in }}}=\left(1-|\Gamma|^{2}\right),
$$

where $\Gamma$ is the reflection coefficient at the transmitting antenna. Note that the impedance match at the transmitting antenna includes the effect of the near-field surroundings and even the presence of the receiving antenna (sensor). Therefore, the impedance-match efficiency is dependent on AUD and the antennas.

The penetration coefficient $p_{T S}$ is defined as the ratio of the received power $P_{\text {rec }}$ by the receiving antenna to the radiated power $P_{\text {trans }}$ from the transmitting antenna:

$$
p_{T S}=\frac{P_{r e c}}{P_{\text {trans }}} .
$$

Therefore, the total penetration coefficient $p_{t o t T S}$ for through-sensing (TS) system can be written as

$$
p_{\text {totTS }}=e_{i m p} p_{T S} .
$$

The total penetration coefficient and the penetration coefficient can be evaluated by either frequency-domain method or time-domain method, as described in the next section.

\section{Evaluation}

\section{A. Time-domain Method}

In time-domain, total penetration coefficient can be calculated as

$$
p_{\text {tot } T S}=\frac{\int_{0}^{T} R_{s}(t)^{2} d t}{\int_{0}^{T} T_{s}(t)^{2} d t},
$$

where $R_{s}(t)$ and $T_{s}(t)$ are the received and input signal pulses in time-domain, respectively. Also $T$ should be chosen properly depending on the applications so that the received signal has reached a steady state. It is obvious that the total penetration coefficient is a pulse-antenna-AUD dependent characteristic, which measures the performance of the antennas and the pulse for a certain AUD. In this paper, the same gaussian input pulse and the same AUD are used for all investigations in order to compare only the antenna performance.

\section{B. Frequency-domain method}

It is not always convenient or available to have time-domain measurements. Therefore, a characterization of the penetration ability can be evaluated in frequency-domain. In frequency domain, it is common to measure $S$ parameters. Then $p_{\text {totT } S}$ and $p_{T S}$ can be evaluated by

$$
\begin{aligned}
p_{f t o t T S} & =\frac{1}{f_{h}-f_{l}} \int_{f_{l}}^{f_{h}}\left|S_{21}\right|^{2} d f \\
p_{f T S} & =\frac{1}{f_{h}-f_{l}} \int_{f_{l}}^{f_{h}} \frac{\left|S_{21}\right|^{2}}{1-\left|S_{11}\right|^{2}} d f,
\end{aligned}
$$

which assumes that the input signal has a uniform spectral density over the bandwidth of $f_{l}-f_{h}$, where $f_{l}$ and $f_{h}$ are the lower and higher ends of the operating frequency band of the UWB sensing system.

\section{Simulation Results}

The AUD in near-field sensing applications is generally a lossy material. The simulations in this work are performed by using CST Microwave Studio [8], where the AUD is constructed of a brick of butter with different thickness of $25 \mathrm{~mm}, 50 \mathrm{~mm}, 75 \mathrm{~mm}$ and $100 \mathrm{~mm}$. This selection is made because of the similarity between permittivity of butter and body fat, and also because of its availability and ease of shaping. The actual average of dielectric properties of the butter over the frequency band was measured using Agilent's $85070 \mathrm{E}$ performance probe [9], [10]. The measured values are

$$
\epsilon_{r}=4.6, \tan \delta=0.04
$$

which are used in the CST simulations.

\section{A. Reflection Coefficient}

The maximum value of the total penetration coefficient is expected to happen when the antenna is touching the AUD. Hence it would be interesting to observe the effect of material on antenna's reflection coefficient. Fig. 3 shows the reflection coefficients of the three antennas in free-space, and while touching lossy blocks of different thickness. It can be seen that the thickness of object in front of antenna has little effect on the reflection coefficient: when the antennas are touching the lossy material, their reflection coefficients do not change very much with the changes in thickness of the object.

On the other hand, as expected, antenna's reflection coefficient when it is touching a lossy material is different from when it is in free space. It is also seen that the degree of these changes depends on the antenna and frequency. It is clear from the plots that the self-grounded Bow-Tie antenna's reflection coefficient, has higher sensitivity to its surroundings compared 
to the other two antennas. At the same time Vivaldi antenna is less sensitive in that case. This dependency should be further investigated for different antennas.

\section{B. Time-Domain Results}

Table I shows the values of $p_{\text {totTS }}$ for the three antennas and various object thickness. Plot of $p_{t o t T S}$ values for antennas versus different object thickness is shown in Fig. 4. The difference in penetration ability of the three antennas at a certain depth is observed. In the case of current input pulse, it is suggested that self-grounded Bow-Tie antenna has an advantage over the other antennas. However, this advantage seems to decrease as the depth is increased.

\section{Frequency-Domain Results}

Tables II and III show the values of $p_{f t o t T S}$ and $p_{f T S}$ for the three antennas and various object thickness, respectively. Plot of $p_{f t o t T S}$ for different antennas versus different object thickness is shown in Fig. 5. The same plot for values of $p_{f T S}$ is shown in Fig. 6. Here we can see the effect of reflected power on the performance of antenna. For example when only the transmitted power $P_{\text {trans }}$ is compared to the received power $P_{\text {rec }}$, Vialdi antenna has advantage over the other two. But if reflected power is taken into account then this advantage is no longer present.

\section{Directivity}

Plots of directivity of aforementioned different antennas are shown in Fig. 7. It is clearly observed that for different antennas the amount of power radiated in the desired direction for near-field application (Penetration ability) is not related to that for far-field application (Directivity).

TABLE I: $p_{t o t T S}$ vs. object thickness

\begin{tabular}{|l|c|c|c|c|}
\hline \multirow{2}{*}{$p_{\text {totTS }}(d B)$} & \multicolumn{4}{|c|}{ Object Thickness $(\mathrm{mm})$} \\
\cline { 2 - 5 } & 25 & 50 & 75 & 100 \\
\hline Antipodal Vivaldi & -13.26 & -17.13 & -20.67 & -23.95 \\
\hline Self-grounded Bow-Tie & -11.22 & -15.85 & -19.97 & -23.71 \\
\hline Vivaldi & -12.25 & -15.46 & -18.19 & -21.05 \\
\hline
\end{tabular}

TABLE II: $p_{f t o t T S}$ vs. object thickness

\begin{tabular}{|l|c|c|c|c|}
\hline \multirow{2}{*}{$p_{\text {ftot } T}(d B)$} & \multicolumn{4}{|c|}{ Object Thickness $(\mathrm{mm})$} \\
\cline { 2 - 5 } & 25 & 50 & 75 & 100 \\
\hline Antipodal Vivaldi & -13.60 & -17.30 & -20.60 & -23.78 \\
\hline Self-grounded Bow-Tie & -13.03 & -17.40 & -21.40 & -25.04 \\
\hline Vivaldi & -15.75 & -18.81 & -21.52 & -24.21 \\
\hline
\end{tabular}

TABLE III: $p_{f T S}$ vs. object thickness

\begin{tabular}{|l|c|c|c|c|}
\hline \multirow{2}{*}{$p_{f T S}(d B)$} & \multicolumn{4}{|c|}{ Object Thickness $(\mathrm{mm})$} \\
\cline { 2 - 5 } & 25 & 50 & 75 & 100 \\
\hline Antipodal Vivaldi & -13.19 & -16.93 & -20.26 & -23.45 \\
\hline Self-grounded Bow-Tie & -12.50 & -16.90 & -20.91 & -24.55 \\
\hline Vivaldi & -15.38 & -18.44 & -21.11 & -23.81 \\
\hline
\end{tabular}

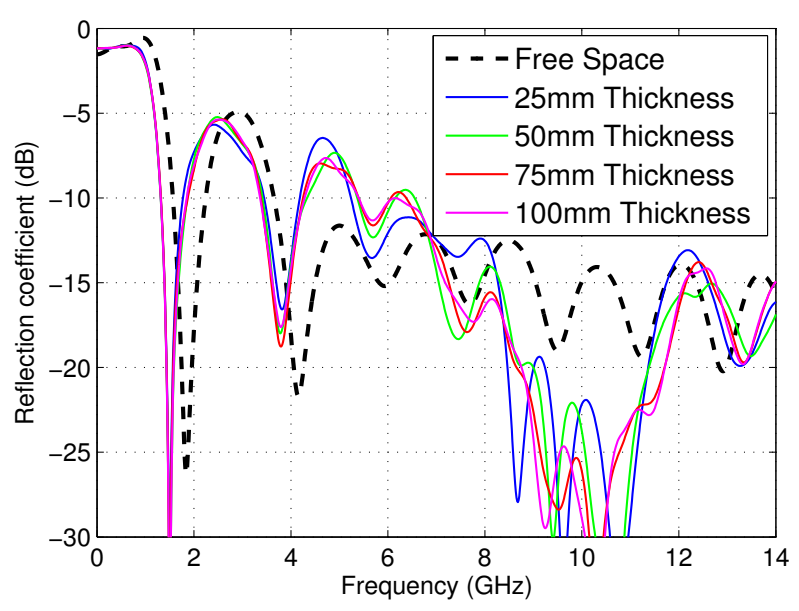

(a) Antipodal Vivaldi antenna

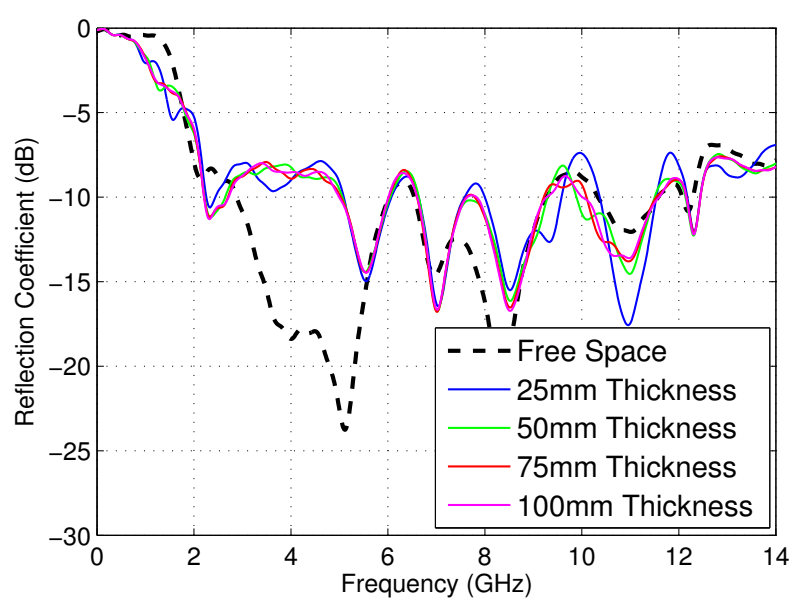

(b) Self-grounded Bow-Tie antenna

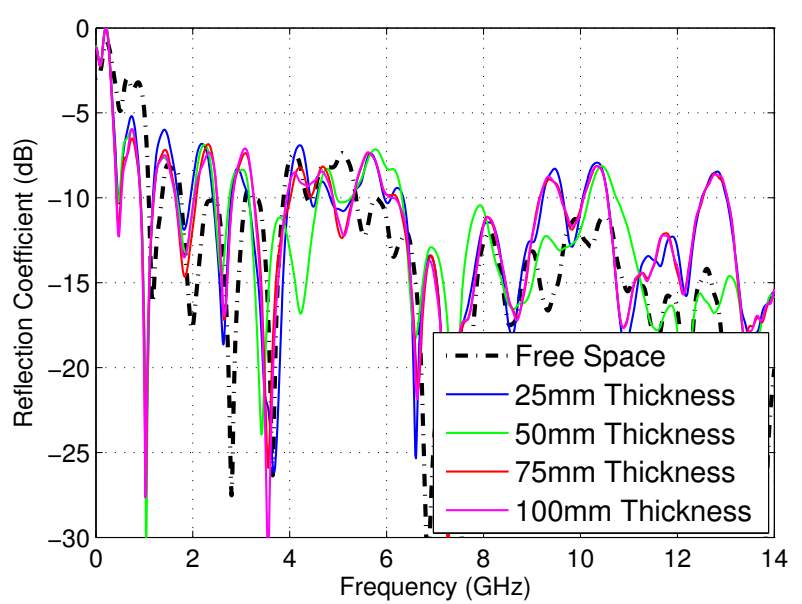

(c) Vivaldi antenna

Fig. 3: Simulated reflection coefficient of the three UWB antennas in free-space and while touching the lossy material with different thickness 


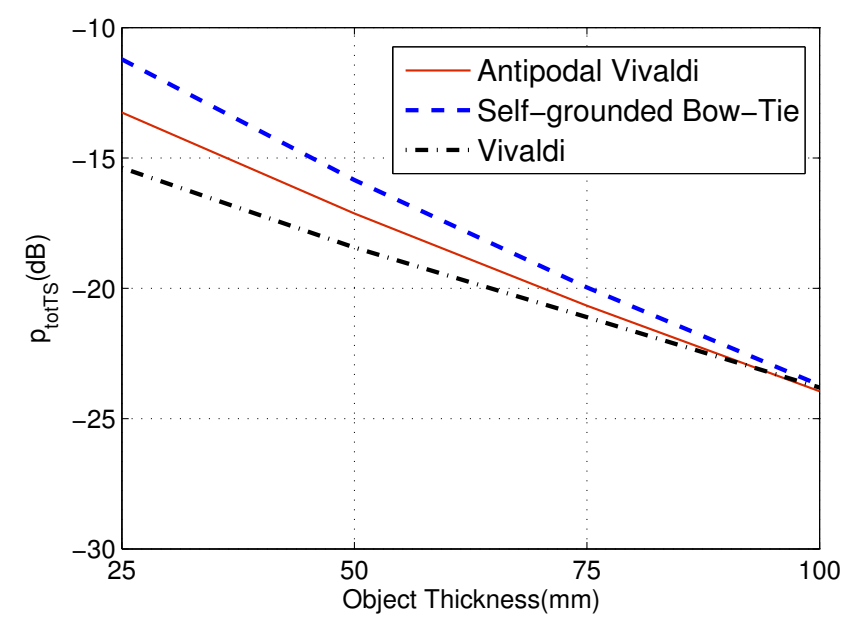

Fig. 4: Simulated $p_{\text {totTS }}$ vs. object thickness

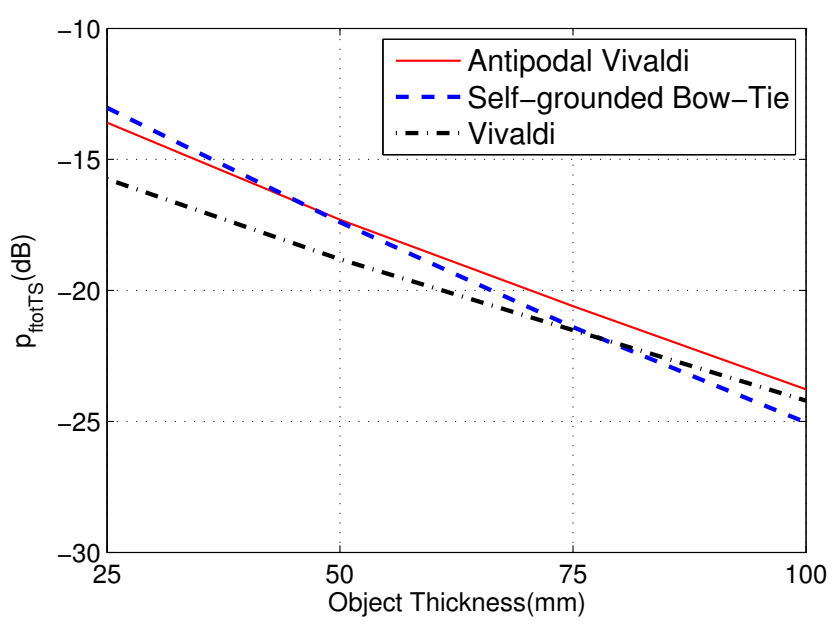

Fig. 5: Simulated $p_{\text {ftotTS }}$ vs. object thickness

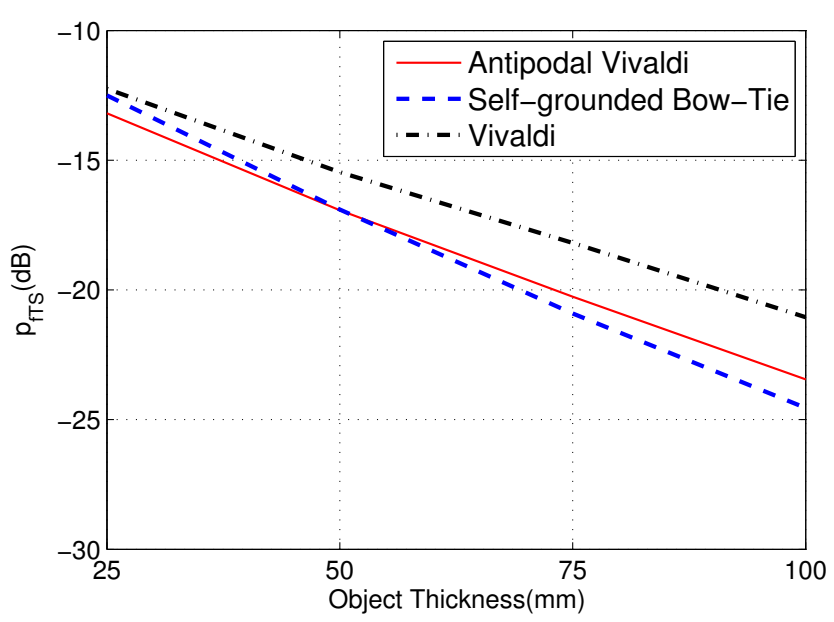

Fig. 6: Simulated $p_{f T S}$ vs. object thickness

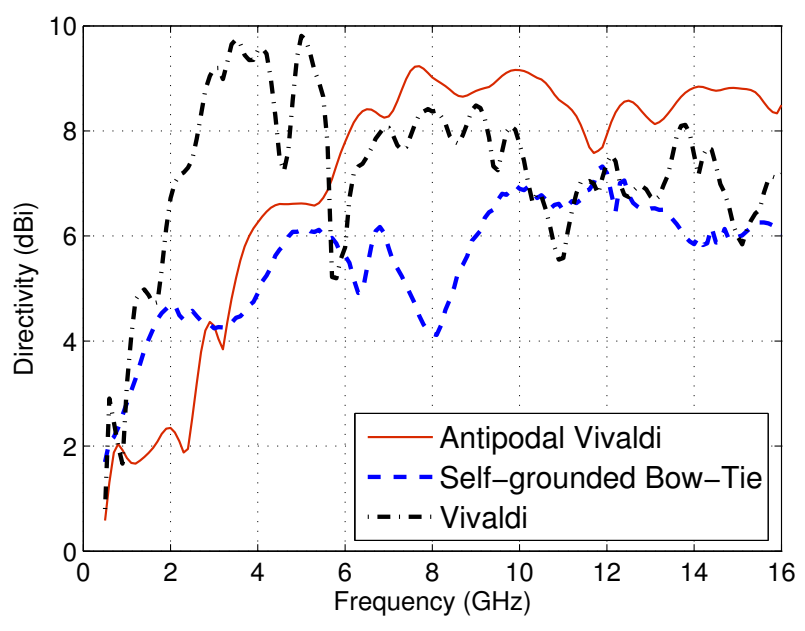

Fig. 7: Directivity of different antennas

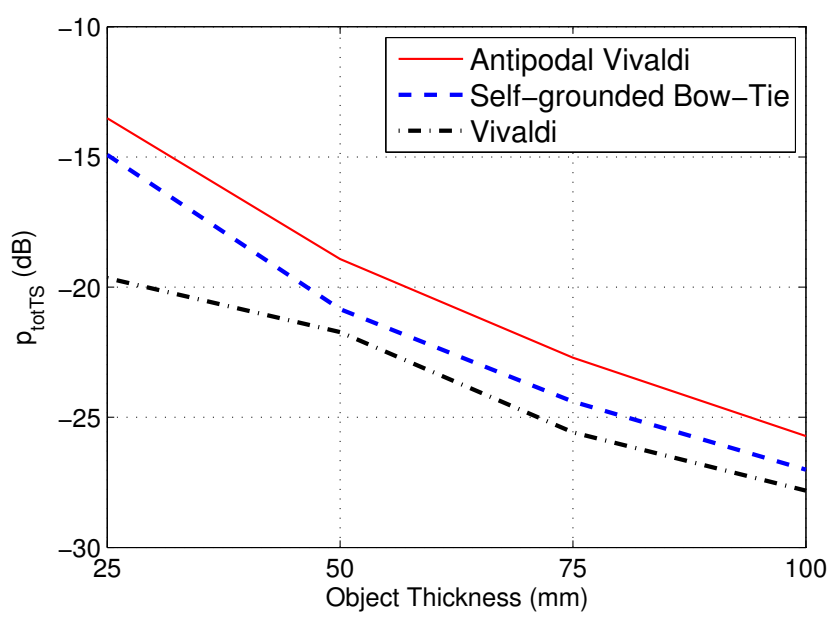

Fig. 8: Measured $p_{t o t T S}$ vs. object thickness

\section{Measurement Results}

\section{A. Time-Domain Results}

Fig. 8 shows the plot of time-domain results for different antennas vs. object thickness. The difference between measurement and simulation results in this case, is due to different input pulse shape applied in measurement compared to the simulation.

\section{B. Frequency-Domain Results}

Figures 9 and 10 show $p_{\text {ftot } T S}$ and $p_{f T S}$, respectively. Here again it can be seen that these results have good agreement with each other and with simulation results. It is interesting to notice that for small thickness the penetration ability of Vivaldi antenna is lower than the other two which have smaller dimensions. But after certain thickness, its penetration ability start to rise above those two. This effect can be explained by higher directivity of Vivaldi antenna due to its size, and transitions from near-field to far-field region. On the other hand 


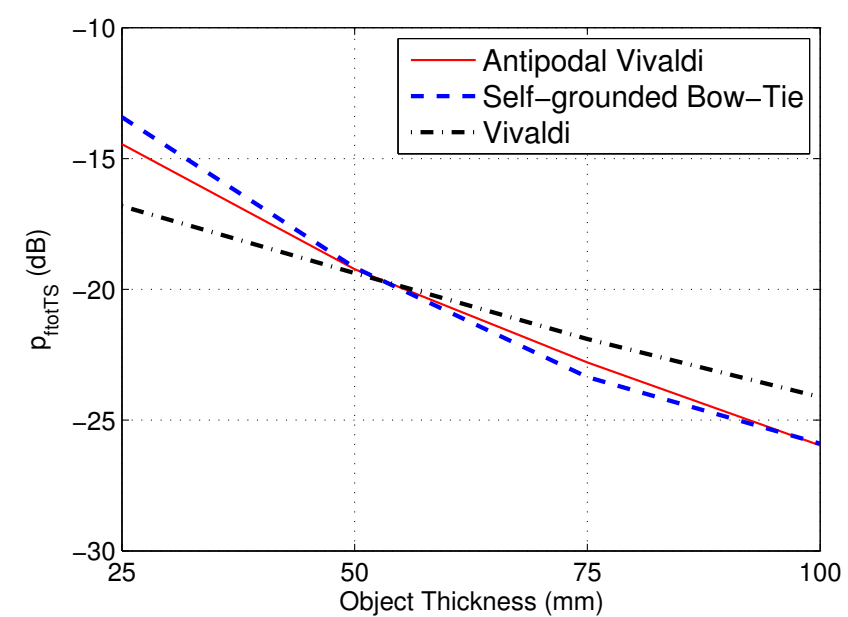

Fig. 9: Measured $p_{f t o t T S}$ vs. object thickness

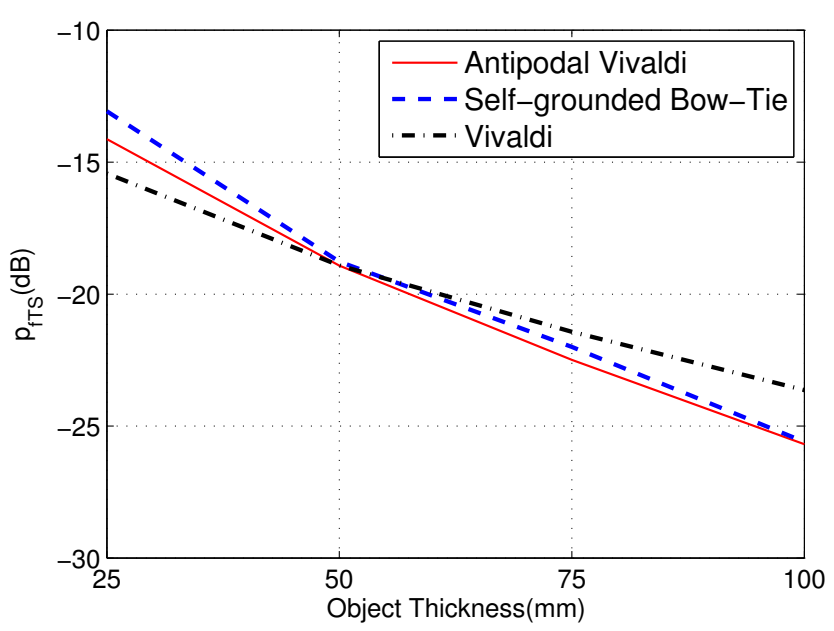

Fig. 10: Measured $p_{f T S}$ vs. object thickness

the difference between frequency-domain and time-domain results is due to non-uniform power spectrum of input pulse.

\section{FUTURE WORK}

This experiment work shows that in near-field sensing scenario, UWB antennas perform in a different manner from that in far-field applications. Therefore, a theoretical study on the topic of maximizing the penetration ability of UWB antennas is undergoing. The theoretical study utilizes a general electromagnetic solver - G2DMULT developed at Chalmers. G2DMULT is a general algorithm based on the S2DS technique that calculates the spectral Greens functions of two-dimensional (2D) multiregion structures by using the method of moments (MoM) for both near-field and far-field problems [11]-[14].

\section{CONCLUSION}

Most antenna characteristics are developed for far-field applications and they are not valid in near-field. A new approach to characterization of antennas in near-field applications was presented in this paper. Penetration ability and its representations in time-domain and frequency-domain have been discussed. It is observed that the amount of power each antenna radiates in desired direction in far-field and nearfield (i.e. Directivity and Penetration ability) are not $100 \%$ correlated. Higher directivity does not always lead to deeper penetration ability in near-field. Hence the choice of antenna in this regard should be according to the application and fieldzone requirement. It seems that for deeper penetration ability, the small size of antenna can be an advantage, since it means that the fields are concentrated in a smaller area. Further investigation on this issue and lower bound for antenna size to achieve deeper penetration ability should be undertaken.

\section{ACKNOWLEDGMENT}

This work has been supported by a Swedish Research Council VR frame project.

\section{REFERENCES}

[1] P.-S. Kildal, Foundations of Antennas, A Unified Approach. Studentlitteratur, 2000.

[2] K. Rosengren and P. Kildal, "Radiation efficiency, correlation, diversity gain and capacity of a six-monopole antenna array for a mimo system: theory, simulation and measurement in reverberation chamber," in $\mathrm{Mi}$ crowaves, Antennas and Propagation, IEE Proceedings-, vol. 152, no. 1. IET, 2005, pp. 7-16.

[3] X. Chen, P. Kildal, J. Carlsson, and J. Yang, "Comparison of ergodic capacities from wideband mimo antenna measurements in reverberation chamber and anechoic chamber," Antennas and Wireless Propagation Letters, IEEE, no. 99, pp. 1-1, 2011.

[4] J. Yang and A. Kishk, "The self-grounded Bow-Tie antenna." Spokane, Washington: 2011 IEEE AP-S International Symp. on Antennas Propag., 3-8 July 2011.

[5] J. Yang and A. A. Kishk, "A novel low-profile compact directional ultrawideband antenna: the self-grounded Bow-Tie antenna," IEEE Trans. Antennas Propagat., vol. 60, no. 3, March 2012.

[6] P. J. Gibson, "The vivaldi aerial," Proc. 9th European. Microwave Conference, pp. 101-105, 1979.

[7] E. Gazit, "Improved design of the vivaldi antenna," in Proceedings IEE Microwaves Antennas and Propagation, April 1988, pp. 89-92.

[8] CST Microwave Studio; http://www.cst.com.

[9] "Technical overview, agilent 85070 e dielectric probe kit $200 \mathrm{MHz}$ to 50 GHz," Agilent Technologies, Inc., May 92003.

[10] "Technical overview, agilent 85071e materials measurement software," Agilent Technologies, Inc., November 62003.

[11] J. Yang and P. S. Kildal, "Presentation of the spectral electric and magnetic field integral equations used in g2dmult for analyzing cylindrical structures of multimaterial regions," Microwave and Optical Technology Letters, vol. 34, no. 2, pp. 88-93, 2002.

[12] J. Yang, U. Carlberg, P. S. Kildal, and M. Kehn, "A fast mode analysis for waveguides of arbitrary cross section with multiple regions by using a spectrum of two-dimensional solutions and asymptotic waveform evaluation," IEEE Trans. Microwave Theory and Techniques, vol. 52, no. 6, pp. 1615-1621, 2004.

[13] J. Yang and P. S. Kildal, "A fast algorithm for calculating the radiation pattern in the longitudinal plane of antennas with cylindrical structure by applying asymptotic waveform evaluation in a spectrum of twodimensional solutions," IEEE Trans. Antennas and Propaga.,, vol. 52, no. 7, pp. 1700-1706, 2004.

[14] — "A fast calculation of impedances of dipoles near cylindrical structure by applying asymptotic waveform evaluation in a spectrum of $2 \mathrm{~d}$ solutions," Microwave and optical technology letters, vol. 43, no. 4, pp. 314-317, 2004. 